\section{Beam quality changes of Gaussian Schell-model fields propagating through Gaussian apertures}

\section{J. Serna, P. M. Mejías, and R. Martínez-Herrero}

The authors are with the Departamento de Óptica, Facultad de Ciencias Físicas, Universidad Complutense, 28040 Madrid, Spain.

Received 25 June 1991. 0003-6935/92/224330-02\$05.00/0.

(1) 1992 Optical Society of America.

Beam quality behavior of Gaussian Schell-model beams that propagate through Gaussian apertures is analyzed.

The control and characterization of the spatial profiles of laser beams is, at present, a field of growing interest because of the attractive possibility of improving the performances of important metrological and industrial processes. Thus, quite recently ${ }^{1,2}$ a family of measurable parameters has been defined in terms of the second-order coherence features of beams that describe their focusing capabilities, divergence properties, and degree of asymmetry or astigmatism under propagation through general $A B C D$ optical systems. Moreover, a new parameter has been introduced in the literature ${ }^{3,4}$ that can be used as an appropriate description of the beam quality in passive and active media. ${ }^{5}$ Among other properties, this characteristic parameter remains invariant under propagation through firstorder systems. An immediate consequence could be derived from this invariance property: in order to improve the quality of an arbitrary beam non- $A B C D$ optical systems are necessarily required. In the present work attention is concentrated on a certain class of soft-edge diffraction apertures, namely, the so-called Gaussian apertures, which, as is seen later, are specially suitable to control the quality of Gaussian Schell-model (GSM) beams. As is well known, these types of field, because of their noncoherent nature, do not produce harmful laser effects, such as speckle or ringing, and, under rather general conditions, they behave as highly directional wave fields. ${ }^{6,7}$ In the following, after the pertinent definitions and the procedure to be used are introduced, we analyze the behavior of the quality of GSM fields that propagate through Gaussian apertures in terms of both their coherence length and their width.

Since GSM beams are partially spatially coherent, it appears appropriate to employ the Wigner distribution function (WDF) formalism. Restricting ourselves, for simplicity, to essentially bidimensional beams, let us then start from the expression of the WDF associated with a GSM field:

$$
\begin{aligned}
h(x, u ; v)= & \left(P_{i} k / \pi\right)\left[\gamma^{2} /\left(1+\gamma^{2}\right)\right]^{1 / 2} \exp \left(-x^{2} / \sigma_{I}^{2}\right) \\
& \times \exp \left\{-k^{2} \sigma_{I}^{2}\left[\gamma^{2} /\left(1+\gamma^{2}\right)\right][u-(x / R)]^{2}\right\} .
\end{aligned}
$$

In this expression $x$ denotes the spatial variable transversal to the propagation direction $z$, $k u$ is the wave vector component along the $x$ axis (hence $u$ would represent an angle of propagation, without taking the evanescent into account), $P_{i}$ is the total irradiance, $\sigma_{I}(z)$ and $R(z)$ are the beamwidth and the curvature radius at the $z$ plane, and the constant $\gamma$ is the so-called global degree of coherence, i.e., the ratio between the transversal coherence length and the beamwidth. It is well known ${ }^{8}$ that integration of the WDF over the angular variable $u$ gives the beam intensity, and its integral over the spatial variable $x$ is proportional to the radiant intensity of the field. Moreover, by defining the average $\langle q\rangle$ in the form

$$
\langle q\rangle \equiv\left(1 / P_{i}\right) \int_{-\infty}^{+\infty} \mathrm{d} x \mathrm{~d} u q h(x, u ; z),
$$

we can introduce a number of characteristic beam parameters, namely, $\langle x\rangle,\langle u\rangle,\left\langle x^{2}\right\rangle$, and $\left\langle u^{2}\right\rangle$, representing, respectively, the center of the beam, its direction of propagation, its (squared) width, and its (squared) far-field divergence (angular spread). Assuming that $\langle x\rangle=\langle u\rangle=0$ (this is not a restriction, since it is simply equivalent to a shift of the coordinate system) a beam quality parameter $Q$ can be defined as follows:

$$
Q=\left\langle x^{2}\right\rangle\left\langle u^{2}\right\rangle-\langle x u\rangle^{2},
$$

where the term $\langle x u\rangle$ vanishes at the beam waist. The lower limit of $Q$ (highest quality) can be shown to be $1 / 4 k^{2}$, reached only by Gaussian beams (GSM fields with $\gamma \rightarrow \infty$ ). For a general GSM beam that propagates through firstorder optical systems we obtain

$$
Q=\left(1 / 4 k^{2}\right)\left(1+1 / \gamma^{2}\right) .
$$

Let us now consider that, at plane $z_{0}$, our GSM field passes through a centered Gaussian aperture defined by its transmittance function

$$
t(x)=\exp \left(-x^{2} / 2 r^{2}\right)
$$

where $r$ represents the aperture width. It is not difficult to show that, after the aperture is crossed, the field remains a GSM type, but its characteristic beam parameters are modified. Their new values at the output of the transmittance are given by the following expressions:

$$
\begin{aligned}
P_{o}= & P_{i} /\left[1+\rho^{2}\left(z_{0}\right)\right]^{1 / 2}, \\
\langle x\rangle_{o}= & \langle u\rangle_{o}=0, \\
\left\langle x^{2}\right\rangle_{o}= & {\left[\sigma_{I}^{2}\left(z_{0}\right) / 2\right] /\left[1+\rho^{2}\left(z_{0}\right)\right], } \\
\langle x u\rangle_{o}= & {\left[\sigma_{I}^{2}\left(z_{0}\right) / 2 R\left(z_{0}\right)\right] /\left[1+\rho^{2}\left(z_{0}\right)\right], } \\
\left\langle u^{2}\right\rangle_{o}= & \left(1 / 2 k^{2}\right)\left\{\left[1+\rho^{2}\left(z_{0}\right)\right] / \sigma_{I}^{2}\left(z_{0}\right)+1 /\left[\sigma_{I}\left(z_{0}\right) \gamma\right]^{2}\right\} \\
& +\left[\sigma_{I}^{2}\left(z_{0}\right) / 2 R^{2}\left(z_{0}\right)\right] /\left[1+\rho^{2}\left(z_{0}\right)\right],
\end{aligned}
$$

where subscripts $o$ and $i$ designate the output and input values, $\sigma_{l}\left(z_{0}\right)$ and $R\left(z_{0}\right)$ refer to the input values, and $\rho\left(z_{0}\right)=$ 

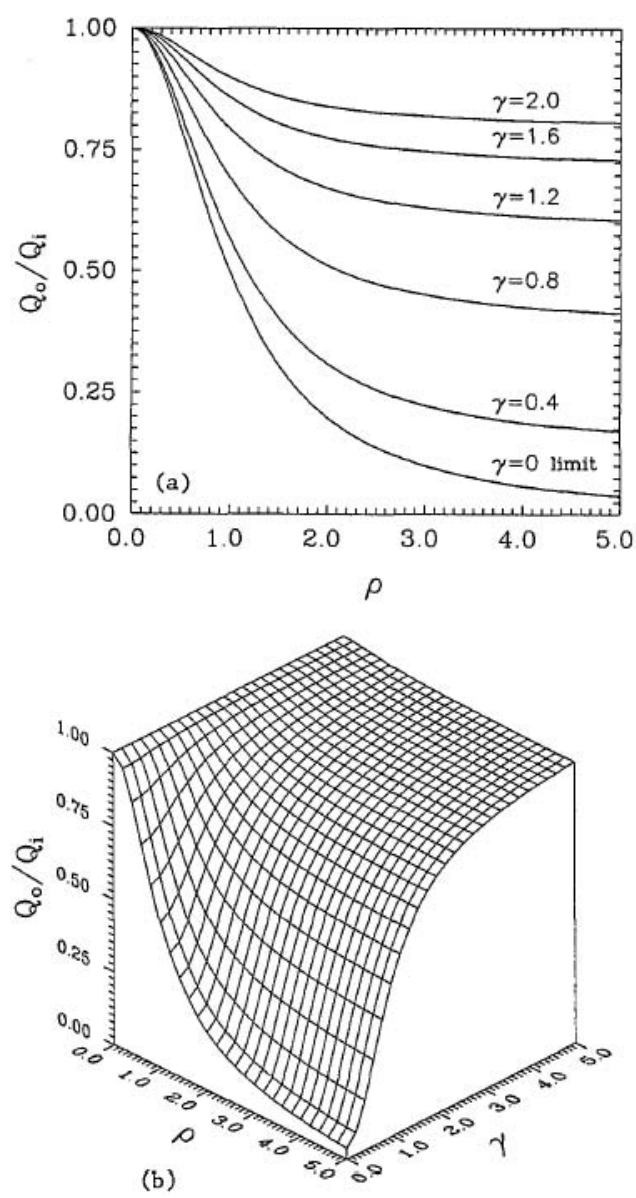

Fig. 1. Quality gain $Q_{o} / Q_{i}$ in terms of $\rho$ and $\gamma$ : (a) parametric representation; (b) tridimensional plot.

$\sigma_{I}\left(z_{0}\right) / r$. From Eq. (6a) it follows that $P_{o}$ does not depend on the coherence properties of the input beam (contained in the parameter $\gamma$ ), and that $P_{o} \leq P_{i}$, with a worst-power ratio $P_{o} / P_{i}$ for smaller values of $r$. Other features can be inferred from Eqs. (6a), (6c), and (6e):

$$
\begin{aligned}
\left\langle x^{2}\right\rangle_{o} /\left\langle x^{2}\right\rangle_{i}= & \left(P_{o} / P_{i}\right)^{2}=1 /\left[1+\rho^{2}\left(z_{0}\right)\right] \\
\left\langle u^{2}\right\rangle_{o}-\left\langle u^{2}\right\rangle_{i}= & 1 / 2 k^{2} r^{2}-\left[\sigma_{I}\left(z_{0}\right) / 2 R\left(z_{0}\right)\right] \rho^{2}\left(z_{0}\right) \\
& /\left[1+\rho^{2}\left(z_{0}\right)\right] .
\end{aligned}
$$

Since $\rho^{2}\left(z_{0}\right)$ is a positive quantity, it is clear from Eq. (7a) that the beamwidth is always reduced after propagation through Gaussian apertures, as expected, and the reduction factor is independent of the coherence properties of the beam. On the other hand, Eq. (7b) expresses the way in which the angular spread of our GSM is modified. Thus, depending on the position (relative to the beam) of the plane $z_{0}$ in which we have placed the transmittance, the far-field divergence could be enlarged or decreased (in fact, by setting the aperture far enough from the waist plane of the input beam, we can reduce the divergence).

Taking into account Eqs. (6c)-(6e), we can readily show that the ratio $Q_{o} / Q_{i}$ takes the form

$$
Q_{o} / Q_{i}=\left\{\gamma^{2}\left[1+\rho^{2}\left(z_{0}\right)\right]+1\right\} /\left\{\left(1+\gamma^{2}\right)\left[1+\rho^{2}\left(z_{0}\right)\right]\right\},
$$

where the input beam quality $Q_{i}$ is given by Eq. (4). We refer to the above ratio as the quality gain ( $Q G)$ of the system. The term gain follows from the fact that smaller values of $Q_{o}$ mean higher beam qualities. It is immediately seen from Eq. (8) that $Q_{o} / Q_{i} \leq 1$. In other words, the beam quality parameter is always improved when a Gaussian aperture is introduced in our $A B C D$ optical system. Equation (8) is plotted in Fig. 1 as a function of parameters $\rho$ and $\gamma$. With fixed $\rho$, the maximum QG is obtained in the incoherent limit $\gamma \rightarrow 0$. On the other hand, for a given $\gamma$, the maximum attainable $Q G$ (smallest value of $Q_{o} / Q_{i}$ ) would be $\gamma^{2} /\left(1+\gamma^{2}\right)$, achieved when $\rho \rightarrow \infty$. Note that parameter $\rho$ depends on $z$ through the quantity $\sigma_{I}\left(z_{0}\right)$. In consequence, Fig. 1 also represents the behavior of the $\mathrm{QG}$ as a function of the relative position $z_{0}$ of the Gaussian aperture with respect to the beam. It is eviednt from this figure that QG would be optimized by placing the transmittance far away from the waist plane of our GSM field. Both the beamwidth and the angular divergence would be reduced. However, in such a case, the total intensity that is transmitted by the aperture would be small. Therefore, in each particular case, the final power requirements of our arrangement will establish the beam quality limit that could be reached by inserting a Gaussian aperture.

In summary, Gaussian apertures can be appropriately used to change the characteristics of GSM fields in a way that depends on the coherence and width of the beam and on the width and relative position of the aperture. At the output the beamwidth is reduced, the far-field divergence is modified, and, more important, the beam quality parameter is always improved.

We thank the Empresa Nacional de Óptica, S.A., and the Comisión Interministerial de Ciencia y Technología of Spain for their support.

\section{References}

1. J. Serna, R. Martínez-Herrero, and P. M. Mejías, "Parametric characterization of general partially coherent beams propagating through $A B C D$ optical systems," J. Opt. Soc. Am. A 8, 1094-1098 (1991).

2. J. Serna, P. M. Mejías, and R. Martínez-Herrero, "Beam quality dependence on the coherence length of Gaussian Schell-model fields propagating through $A B C D$ optical systems," J. Mod. Opt. 39, 625-635 (1991).

3. S. Lavi, R. Prochaska, and E. Keren, "Generalized beam parameters and transformation laws for partially coherent light," Appl. Opt. 27, 3696-3703 (1988).

4. R. Simon, N. Mukunda, and E. C. G. Sudarshan, "Partially coherent beams and a generalized $A B C D$-law," Opt. Commun. 65, 322-328 (1988).

5. R. Martínez-Herrero and P. M. Mejías, "Beam characterization through active media," Opt. Commun. 85, 162-166 (1991).

6. E. Collet and E. Wolf, "Is complete spatial coherence necessary for the generation of highly directional light beams?" Opt. Lett. 2, 27-29 (1978).

7. J. Deschamps, D. Courjon, and J. Bulabois, "Gaussian Schellmodel sources: an example and some perspectives," J. Opt. Soc. Am. 73, 256-261 (1983).

8. M. J. Bastiaans, "Application of the Wigner distribution function to partially coherent light," J. Opt. Soc. Am. A 3, 12271246 (1986). 